\title{
Cerámica y vibración de la luz. Nuevas técnicas de nacarado e irisado y caracterización colorimétrica
}

\section{Ceramics and reflected light new pearlescent and iridescent techniques and colorimetric characterization}

\author{
$\underline{\text { V. Echarri Iribarren }}^{(*)}$, A. B. González Avilés ${ }^{(*)}$, M. Ródenas ${ }^{(* *)}$, J. Olivares ${ }^{(* *)}$
}

RESUMEN

Los materiales cerámicos han abierto nuevos espacios de innovación en su aplicación en las envolventes de los edificios en los últimos años. Sin embargo, ha sido escasa la investigación en su relación con la vibración de la luz, la percepción visual y su integración en el paisaje. En esta investigación se estudia la producción y aplicación de piezas de gres porcelánico en un auditorio. El uso de un recubrimiento cerámico con un acabado irisado-nacarado con reflejo metálico responde a la intención de generar una piel vibrante en constante cambio frente a la luz natural y el entorno. Se ha diseñado un proceso de vitrificado y deposición de metales, a través de tres cocciones sucesivas. Se analizan las propiedades del esmaltado y los factores de microfisuración que refuerzan el irisado. Se ha hecho un análisis colorimétrico evaluando los colores goniocromáticos o iridiscentes, midiendo el factor de radiancia espectral de la luz.

Palabras clave: gres porcelánico; irisado-nacarado; deposición de metales; reflectancia; colores goniocromáticos; zirconio; titanio.

\section{ABSTRACT}

Recent years have witnessed the development of innovative applications for ceramic materials as building envelopes. However, little research has been conducted on the relationship between these materials and reflected light, visual perception and integration in the landscape. The aim of the present research was to study the production and application of porcelain stoneware panels in an auditorium. The idea behind using a ceramic coating with an iridescent, pearlescent finish and a metallic sheen was to create a vibrant, constantly changing surface that reflected the natural light and the environment. We designed a metal deposition and glazing process based on three successive firings, and analysed the glaze properties and microcracking factors that reinforced the iridescent lustre. We also performed a colorimetric analysis to assess the goniochromatic or iridescent colours, measuring the spectral radiance of the light.

Keywords: porcelain stoneware; iridescent-pearlescent; metal deposition; reflectance; goniochromatic colours; zirconium; titanium.

(*) Universidad de Alicante (España).

(**) COR Asociados.

Persona de contacto/Corresponding author: Victor.Echarri@ua.es (V. Echarri Iribarren)

Cómo citar este artículo/Citation: Echarri Iribarren, V., González Avilés, A. B., Ródenas, M., Olivares, J. (2016). Cerámica y vibración de la luz. Nuevas técnicas de nacarado e irisado y caracterización colorimétrica. Informes de la Construcción, 68(544): e164, doi: http:// dx.doi.org/10.3989/ic.15.162.m15.

Copyright: (C) 2016 CSIC. Licencia / License: Salvo indicación contraria, todos los contenidos de la edición electrónica de Informes de la Construcción se distribuyen bajo una licencia de uso y distribución Creative Commons Attribution License (CC BY) Spain 3.o. 


\section{INTRODUCCIÓN}

Desde la década de los ochenta la cerámica ha ido recuperando el espacio perdido que durante tantos siglos le había correspondido. Tras una época de reclusión al baño y cocinas, la industria cerámica ha sabido renovarse transformando todos sus procesos y recuperando la innovación y tecnología características del sector, disipadas desde finales del siglo xIx. Esta recuperación ha propiciado una mayor competitividad y un mayor desarrollo de soluciones constructivas que han sabido adaptar el material cerámico a las nuevas necesidades funcionales, estéticas y económicas que la arquitectura demanda de ella. Atrás quedan los años de estancamiento tecnológico y la neutralidad de sus productos. Desde hace 15 años se está viviendo una auténtica revolución de la cerámica gracias a una elevada inversión en el proceso de fabricación unido a una elevada calidad en el diseño de producto.

En esa alianza entre el diseño y la fabricación los arquitectos han sabido ver un potencial de desarrollo creativo, artístico y proyectual. No es casualidad que en estas dos últimas décadas hayan aparecido numerosos edificios con soluciones innovadoras de aplicación de la cerámica. Detrás de todos ellos hay siempre una alianza fundamental entre estudios de arquitectura y artesanos o fabricantes.

La cerámica se ha consolidado como un material polifacético (1) que permite una oferta de recubrimientos amplísima gracias a la investigación tanto en el proceso de fabricación como en los procesos de post-fabricado que permite. Ello ha propiciado una libertad compositiva que ha dado rienda suelta a la creatividad y que podemos observar en gran parte de los premios de arquitectura de nuestro país y fuera de él. ¿Cuándo podemos datar esta nueva situación del sector cerámico? Es una respuesta difícil atendiendo al proceso industrial, sin embargo, no lo es tanto si estudiamos esa reconocida y afamada producción arquitectónica.

Atendiendo al proceso industrial la doctora Vacca sitúa el punto de inflexión en el rol protagónico del sistema de cocción y en la revolución productiva tras la inclusión de los hornos monoestratos y la llegada del gas natural a la Comunidad Valenciana, 1980-1993 (2). Durante este mismo periodo aparecen innovaciones de los procesos de molienda de arcillas por vía húmeda y atomizado, aparece la industria auxiliar del tercer fuego, se incorporan al proceso nuevos medios de impresión, corte, etc. El desarrollo de todos estos procesos coincidió con los primeros años de formación de importantes instituciones oficiales como ITC, AICE, ALICER, ANFFECC, en colaboración con empresas del sector cerámico y con ASCER. Una vez incorporados estos nuevos procesos y con una enorme competitividad durante la década 1994 a 2004, España se sitúa por delante de Italia en producción. Sin duda, esta década genera los cimientos necesarios para el dominio de la técnica al servicio del proyecto y mejora notablemente las técnicas de colocación.

En este último periodo se recupera la tradición artesanal olvidada desde la arquitectura modernista. Si entonces los arquitectos diseñaban piezas cerámicas artesanales, fabricadas con materiales y sistemas tradicionales ex profeso, ahora esta misma circunstancia se apoya en todo el avance tecnológico, que afecta tanto al material como a la informatización de los procesos de fabricación, decoración y moldeado.

\section{INVESTIGACIÓN E INNOVACIÓN: CERÁMICA Y ARQUITECTURA}

Un ligero repaso de la producción arquitectónica con empleo de soluciones cerámicas innovadoras, permite comprobar el interés de los estudios de arquitectura por el empleo del material y una mayor indagación en su proceso de fabricación. Mies Van der Rohe explicaba la manera en que los arquitectos piensan el modo correcto de utilizar los materiales frente a pensar en la forma, y cómo posteriormente es aceptado el resultado (3). En los ejemplos de aplicaciones que aquí se presentan y concretamente en el objeto de estudio, se entiende que esta circunstancia no es lineal. Es precisamente en la no aceptación de los resultados y la intervención en el proceso de fabricación, donde la relación entre arquitectura y el sector cerámico ha dado sus mejores frutos.

La brillante etapa de relación entre Cerámica y Arquitectura se puede acotar temporalmente en la última década de producción arquitectónica. Verdaderamente es a principios del siglo xxi cuando empezamos a tener los primeros ejemplos representativos de un uso singular de la cerámica. Así, encontramos entre los años 2004 y 2005 el Mercado de Santa Caterina de Enric Miralles, el Pabellón de España en Aichi de Alejandro Zaera y el Palacio de Congresos de Peñíscola de Nieto y Soberano (4). Estos tres ejemplos permiten hablar de una de las propiedades más importantes, que se repetirá año tras año en los diferentes ejemplos expuestos: la variedad de soluciones por el tipo de técnica de conformado de la cerámica empleada. Mientras que en el caso de Peñíscola la cerámica utilizada en fachada se realizó mediante conformado manual, en Barcelona Miralles realizó la cubierta de Santa Caterina con una cerámica prensada en seco, y en Aichí, Zaera conformó la envolvente del pabellón con elementos cerámicos fabricados mediante un prensado plástico. Obras como el pabellón de España de la Expo de Zaragoza, con la distinción del Premio MIES VAN DER ROHE 2009, Villa Nurbs (5) como paradigma de los procesos de fabricación digital, y el paseo de la playa de Poniente de Benidorm de Carlos Ferrater - premio FAD de Arquitectura- corroboran la excelente relación de la cerámica y la arquitectura. Estos tres ejemplos permiten distinguir claramente no solo la variedad de los proyectos a los que permite acceder el uso del material, sino también lo camaleónico de su empleo en cuanto al factor económico de su diseño o puesta en obra.

José Durán describía la situación del material cerámico en 2007 como un material que había tocado fondo ante la opresión de los procesos de producción, el culto a la cosmética en la sociedad moderna y la desaparición de la figura del artesano como puente entre el mundo de las ideas y la técnica (6). La relación entre Arquitectura y Cerámica es en este momento tan palpable que los arquitectos más reconocidos internacionalmente tensan hasta el límite el empleo del material. Valga como ejemplo que Renzo Piano está trabajando paralelamente en paneles prefabricados de gres esmaltado en la fachada del Complejo Central Saint Giles, Londres, Reino Unido.

Queda mucho por hacer en este nuevo proceso de ideación arquitectónico-cerámica. En muchas ocasiones la falta de tiempos más amplios no permite incorporar a determinados procesos un mayor control y ensayo de las soluciones en laboratorio. O tal vez sea más apropiado decir un mayor control de los acabados frente a soluciones simplemente geométricas o de color. La investigación desarrollada en este artículo nos permite ver de qué forma podemos intervenir en el proceso de fabricación de un material cerámico para evaluar y contro- 
lar las propiedades de reflexión, vibración y control de la luz solar y artificial sobre las piezas cerámicas en el caso de envolventes de edificios. Antes de continuar es necesario incidir en que se ha realizado escasa investigación en las propiedades de reflexión, vibración y control de la luz solar y artificial sobre las piezas cerámicas en el caso de envolventes de edificios. Éste es el objetivo principal de este artículo.

Para ello, entre los edificios anteriormente mencionados, se ha elegido el auditorio de la Algueña, obra de Jesús Olivares y Miguel Ródenas del estudio alicantino COR Asociados, por ser el que desarrolla una mayor investigación en las características visuales y la percepción del edificio gracias a la intervención en su envolvente. El proyecto objeto de estudio de este artículo es un claro ejemplo de optimización de recursos económicos para un resultado arquitectónico brillante, con un presupuesto de $776.885 €$ para $1.020 \mathrm{~m}^{2}$ construidos.

\section{AUDITORIO DE LA ALGUEÑA. CERÁMICA Y REFLEXIÓN DE LA LUZ}

El proyecto del auditorio y Casa de la Música de Algueña (MUCA) se planteó a sus habitantes como una oportunidad de construir un hito reconocible capaz de quedar en la memoria de los visitantes, y convertirse en seña de identidad. $\mathrm{Su}$ planteamiento se basa en dos supuestos. El primero conseguir restaurar los $670 \mathrm{~m}^{2}$ del antiguo cuartel de la Guardia Civil introduciendo en su intrincada estructura portante todo el programa requerido. Y el segundo, construir una caja que albergara el auditorio con 230 butacas y sala polivalente, un total de $350 \mathrm{~m}^{2}$ construidos.

Fue este segundo supuesto el que llevó a proyectar un edificio lleno de vibración de luz y color, con una rotundidad volumétrica capaz de interactuar y redefinir su entorno inmediato, ya sea por cambios en la iluminación o por movimiento del observador, estableciendo con ello una relación paisajística con las canteras de mármol crema marfil coto. Lo vibrante, cambiante, efectista del proyecto fue condición primaria en el proceso de diseño. El objetivo era un edificio cuya percepción fuera distinta cada vez que las condiciones de contorno se modificaran, por ejemplo, cada vez que una nube se moviera, o en función de la hora a la que se contemplara (Figura 1). De ahí que se iniciara un proceso de investigación del efecto iridiscente nacarado producido en piezas de gres porcelánico como acabado de las fachadas del auditorio. Un acabado ca-
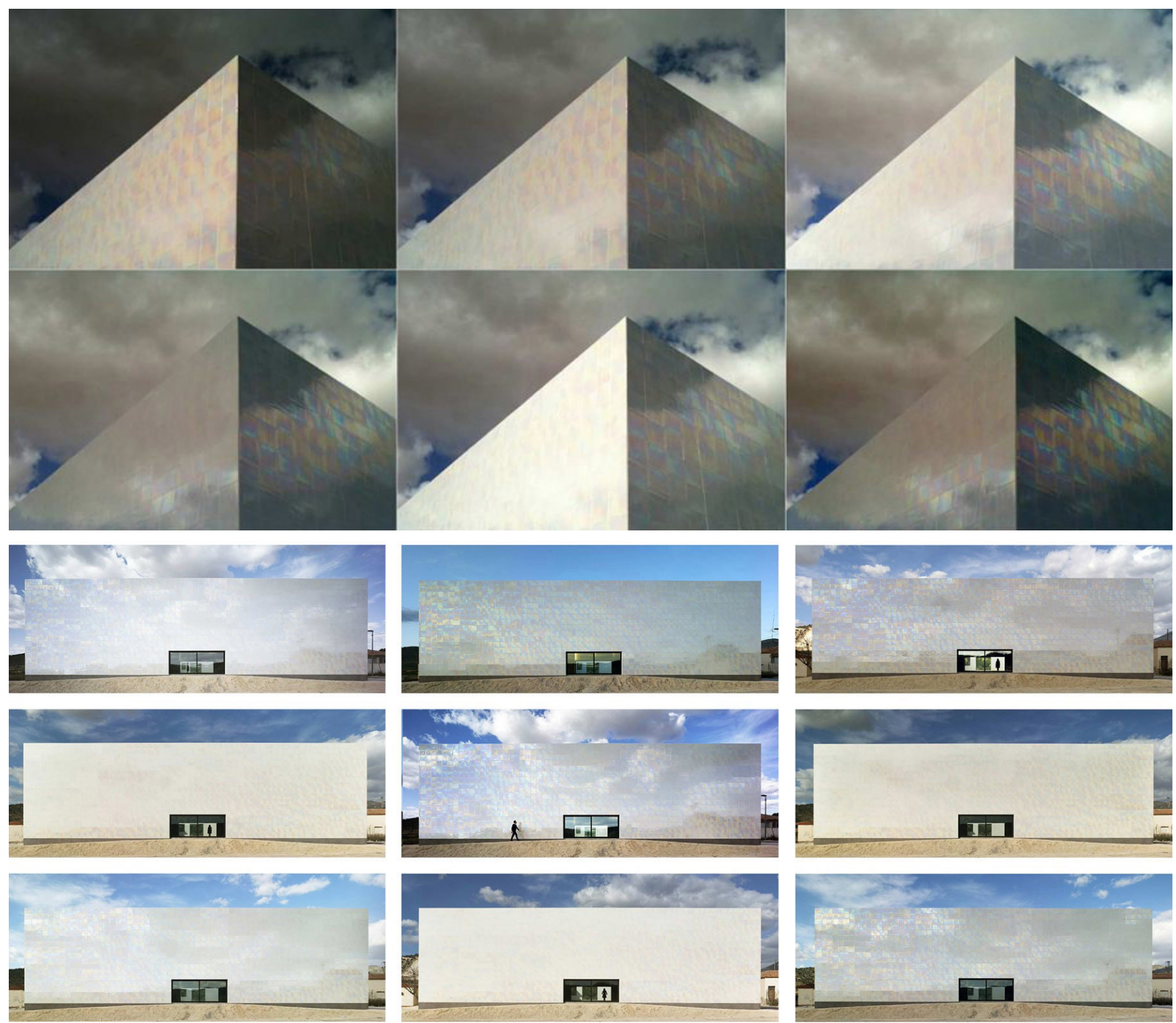

Figura 1. Fotografía de la fachada del MUCA. (C) David Frutos. 
paz de reproducir las exigencias de diseño requeridas y que, al no encontrarse en el mercado, ha generado un nuevo producto, «Iridescent», comercializado por el estudio de arquitectura COR \& Asociados.

El material debía contar, entre sus cualidades funcionales, con un bajo índice de porosidad, gran resistencia a heladas, alta resistencia al cuarteo y alta resistencia a flexión. Dado el bajo presupuesto de la obra, el gres porcelánico se postuló como uno de los materiales principales por aglutinar tanto las características de resistencia y bajo coste de mantenimiento como la susceptibilidad de obtener las condiciones estéticas de iridiscencia y nacarado. Las piezas de $60 \times 60 \mathrm{~cm}$ se disenaron y produjeron ex novo para este proyecto.

Frente a los ejemplos de arquitectura y cerámica antes descritos, la apuesta por el uso del material cerámico con la aplicación de técnicas existentes de cocción, vitrificado y deposición de metales, que añade esa apariencia nacarada al material, responde a la decisión de no generar una situación tectónica ni formal, sino perceptiva.

\section{DESCRIPCIÓN DEL PROCESO DE FABRICACIÓN}

El cerámico Iridescent (iridiscente-porcelánico) empleado en el auditorio de la Algueña es un material desarrollado sobre pastas cerámicas gresificadas a altas temperaturas. Mediante las técnicas que se describen a continuación se consiguen las cualidades técnicas y la calidad que se exige en el campo de la arquitectura para revestimientos especiales.

El material se realiza mediante una producción semiartesanal. La técnica usada proviene de la cerámica de reflejos metálicos del tercer fuego, cuyos orígenes se remontan al siglo IX (7). Cada una de las piezas de revestimiento en fachada es prensada en vía seca por sistema unidireccional en prensas de efecto simple, donde la presión se realiza sólo en una de las superficies de la pieza. El método es sencillo y facilita su automatización, lo que permite alcanzar producciones de mayor rendimiento que con otros tipos de prensado.

A continuación las piezas recién moldeadas se introducen en un secadero continuo para reducir su humedad, duplicando o triplicando así su resistencia mecánica y permitiendo así su posterior procesado. En una fase posterior se aplica el esmalte que permita alcanzar un nivel de brillo y tersa textura para el desarrollo de ese efecto iridiscente, nacarado y metalizado (Figura 2). Al tratarse de un proceso cerámico con intervención de materiales con puntos de maduración diferentes, el orden de las cocciones aplicadas debe ir de mayor a menor, excepto en los pre-bizcochados de los greses. Éstos arrancan con bizcochados que permiten eliminar cierta materia orgánica si la hubiera, o simplemente facilitar los procesos de manipulación y esmaltado, sobre todo cuando se trata de piezas especiales, tanto por la fragilidad de su estructura como por su tamaño.

La consecución del efecto iridiscente se lleva a cabo en un proceso de tres cocciones, e incluso cuatro, según el caso: la primera es a $950^{\circ} \mathrm{C}$ para bizcocharla, la segunda es para cocer el esmalte base blanco, y vitrificar el bizcocho a $1.180^{\circ} \mathrm{C}$ en ciclo rápido, y la última la que consigue multiplicar el efecto irisado-nacarado o reflejo metálico entre 650 y $780^{\circ} \mathrm{C}$, pudiendo ser ésta oxidante o reductora. Es precisamente antes de esta tercera cocción cuando se aplica sobre los esmaltes vitrificados el bálsamo formado por disoluciones de sales metálicas en resinatos u otros vehículos según a qué tipo de cocción se someta a la pieza (8). Siguiendo pautas de recientes investigaciones se realizaron diversas combinaciones eutécticas (9), (10), (11), que permiten alcanzar unos resultados estructurales satisfactorios.

El recubrimiento se compone de una mezcla de óxidos de diversos metales, con partículas de diferentes coeficientes de dilatación, y tamaños de partícula comprendidos entre 50 y $35^{\circ}$ micras. Éstos llegan a presentar cambios de fase durante la etapa de cocción. Las investigaciones de Palacios y Otamendi en el cuerpo de entrada del Palacio de Maudes de Madrid, empleaban azulejos de base blanca con tonos dorados, granates y malvas por los esmaltes opacos con una base fundamentalmente de estaño como elemento opacificador y óxidos alcalinos (sodio y potasio) (12). En algunas investigaciones recientes se han descrito efectos de irisado y nacarado aplicando esmaltes que contenían diversos tipos de óxidos metálicos: óxido de cinc, óxido de zirconio, óxido de itrio, óxido de titanio, óxido de hafnio y óxido de cerio (13). Se han realizado diversas combinaciones de ellos, siendo menos frecuente la presencia de óxido de hafnio y óxido de cerio. Así se desprende además del análisis de la composición de las partículas analizadas.

En el caso que nos ocupa, a las piezas de gres porcelánico Iridescent se aplicaron esmaltes compuestos por óxido de
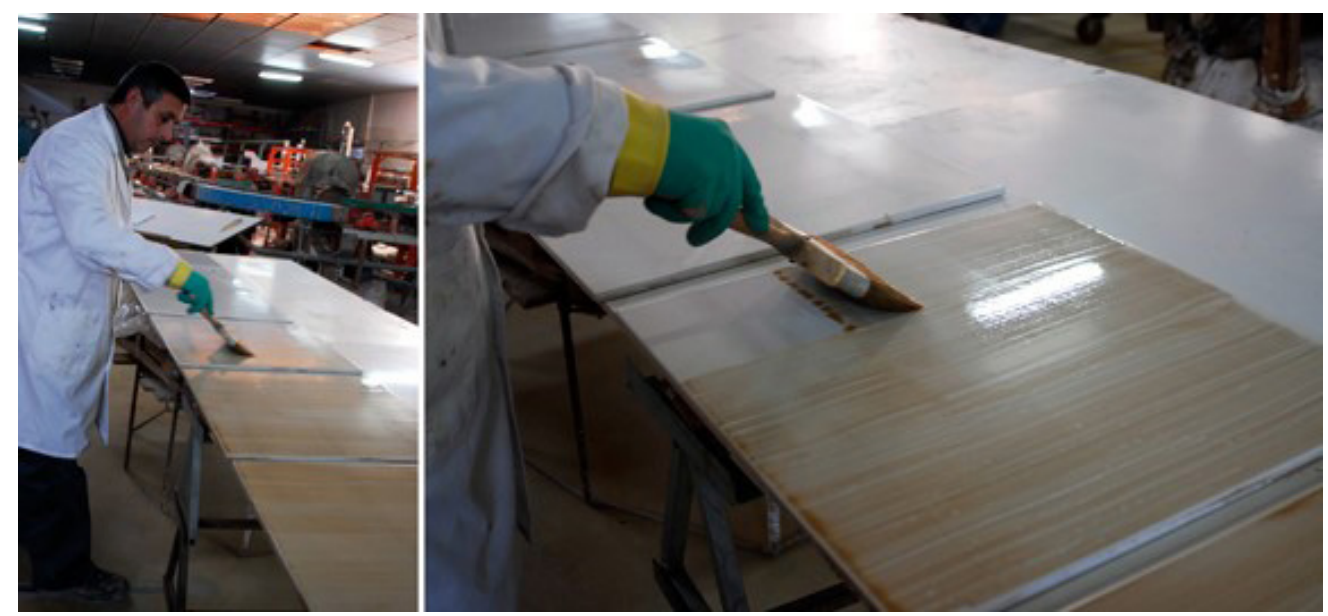

Figura 2. Fotografía del proceso manual de imprimación del cerámico. 


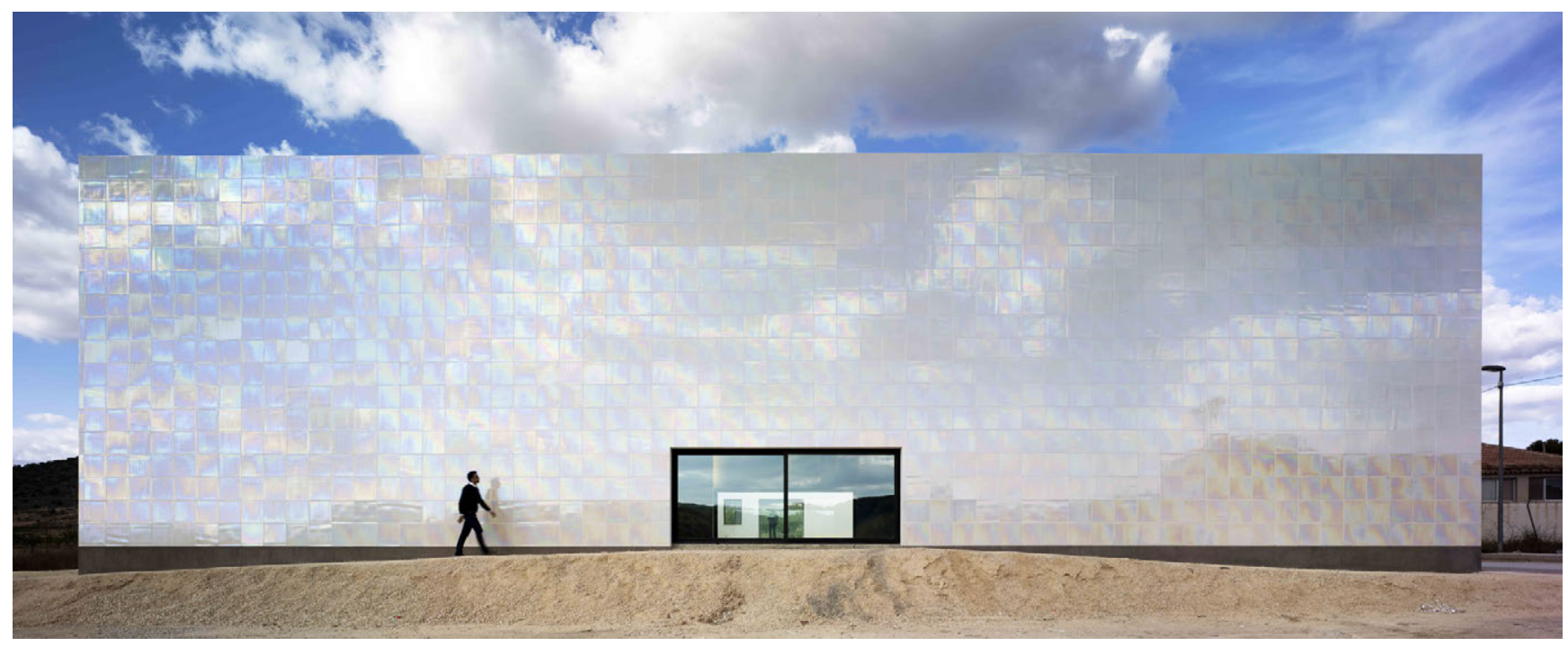

Figura 3. Auditorio de la Algueña. Fachada principal. Efecto de reflexión de la luz.

zirconio, óxido de cinc y óxido de titanio. Las partículas son esferas cerámicas con contenido en óxido de circonio mayor del $50 \%$ en peso, esferas cerámicas de óxido de circonio y de óxido de titanio o mezclas de ellas. Las mezclas se presentan mediante suspensión en medio orgánico o inorgánico, y se aplican sobre los cuerpos cerámicos manualmente mediante brocha para su posterior cocción en un horno de cocción rápida. La aplicación mediante cualquiera de los métodos habituales, campana, aerógrafo, esmaltado a discos, pulverización, inmersión, aplicaciones serigráficas o aplicaciones en seco estuvieron presentes en el proceso de generación del cerámico iridiscente. Sin embargo, se descartaron al buscar una producción artesanal que asegurara la inexistencia de dos acabados exactamente iguales. De esta forma, aplicando el esmalte con brocha, se logró además reforzar el efecto iridiscente de las piezas de gres porcelánico (Figura 2).

El proceso fue desarrollado de forma muy esmerada para poder obtener los resultados deseados. Éstas son básicamente, y de forma resumida, las fases de producción de las piezas de gres porcelánico Iridescent:

- Fase 1. Bizcochado de pieza de gres porcelánico de $60 \times 60$ $\mathrm{cm}$ con cocción a $950^{\circ} \mathrm{C}$. Fabricación industrializada por prensado. Permite eliminar la presencia de materia orgánica.

- Fase 2. Aplicación de esmalte base blanco para vitrificar el bizcocho. Cocción a $1.180{ }^{\circ} \mathrm{C}$. Se obtienen piezas iniciales con esmalte en base blanco para fases posteriores. El proceso de las fases 1 y 2 se podrían hacer también en monococción.
- Fase 3. Aplicación de esmalte a base de óxido de titanio, óxido de zirconio y óxido de zinc. Se aplicaría con brocha de forma manual. Cocción a $1.180^{\circ} \mathrm{C}$. Se producen resultados iniciales de iridiscencia y nacarado.

- Fase 4. Aplicación de esmalte a base óxido de titanio principalmente. Recubrimiento final entre 0,5 y 1 micra. Cocción a temperatura entre 650 y $780^{\circ} \mathrm{C}$. Se produce un cuarteado de esta última capa de vitrificado que multiplica el efecto iridiscente de la reflexión de la luz, con colores y tonalidades de colores.

Se han recogido cinco muestras del desarrollo descrito para la fabricación de las piezas definitivas (Figura 4). Durante dicho proceso fueron modificándose los metales añadidos al bálsamo para conseguir los brillos y colores del irisado. Hemos procedido a caracterizar el resultado del vitrificado multicapa de las 5 piezas cerámicas. Se han realizado análisis de difracción de rayos X (DRX) y microscopía electrónica de barrido. La DRX se hizo en dos fases, para ángulo $2 \theta$ entre 25-113 y 3-70, y step 0,05. En la Gráfica 1, correspondiente a la muestra n. ${ }^{\circ} 3$, se muestra la presencia de silicato de zirconio $\mathrm{Zr}\left(\mathrm{SiO}_{4}\right)$, mientras que en la muestra $n .{ }^{\circ} 5$, fase final del proceso, se detecta la presencia de anatasa $\mathrm{TiO}_{2}$ y silicato de zirconio $\mathrm{Zr}\left(\mathrm{SiO}_{4}\right)$.

Los resultados de la microscopía electrónica de barrido confirman los anteriores resultados. En la Figura 5 se observa a 300 aumentos la presencia de metales en el vitrificado. Son principalmente zirconio, zinc y titanio. Mientras que los dos últimos tienen un reparto homogéneo en la superficie, el zirconio se concentra en algunas zonas (Figura 6). La cristaliza-

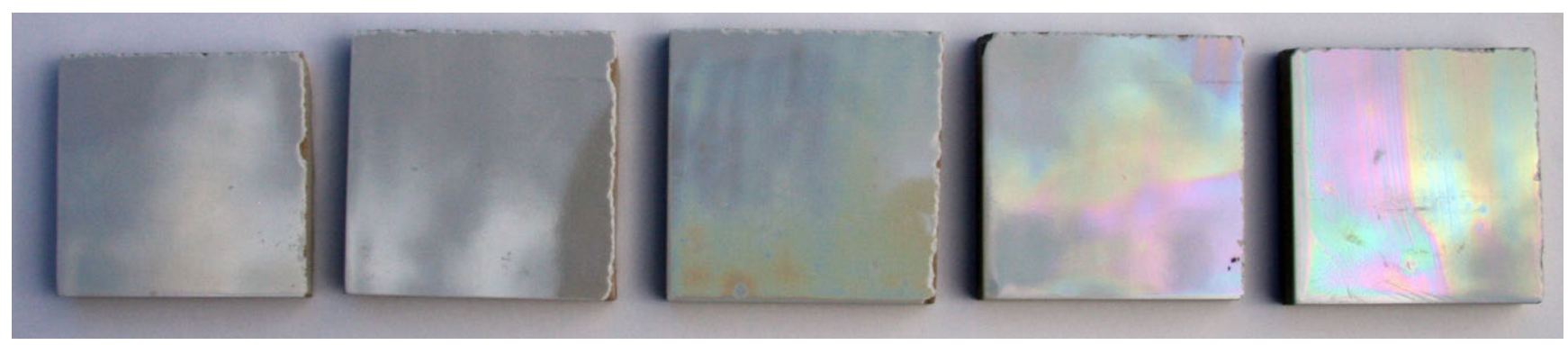

Figura 4. Fotografía de las 5 muestras de gres porcelánico esmaltado analizadas. 


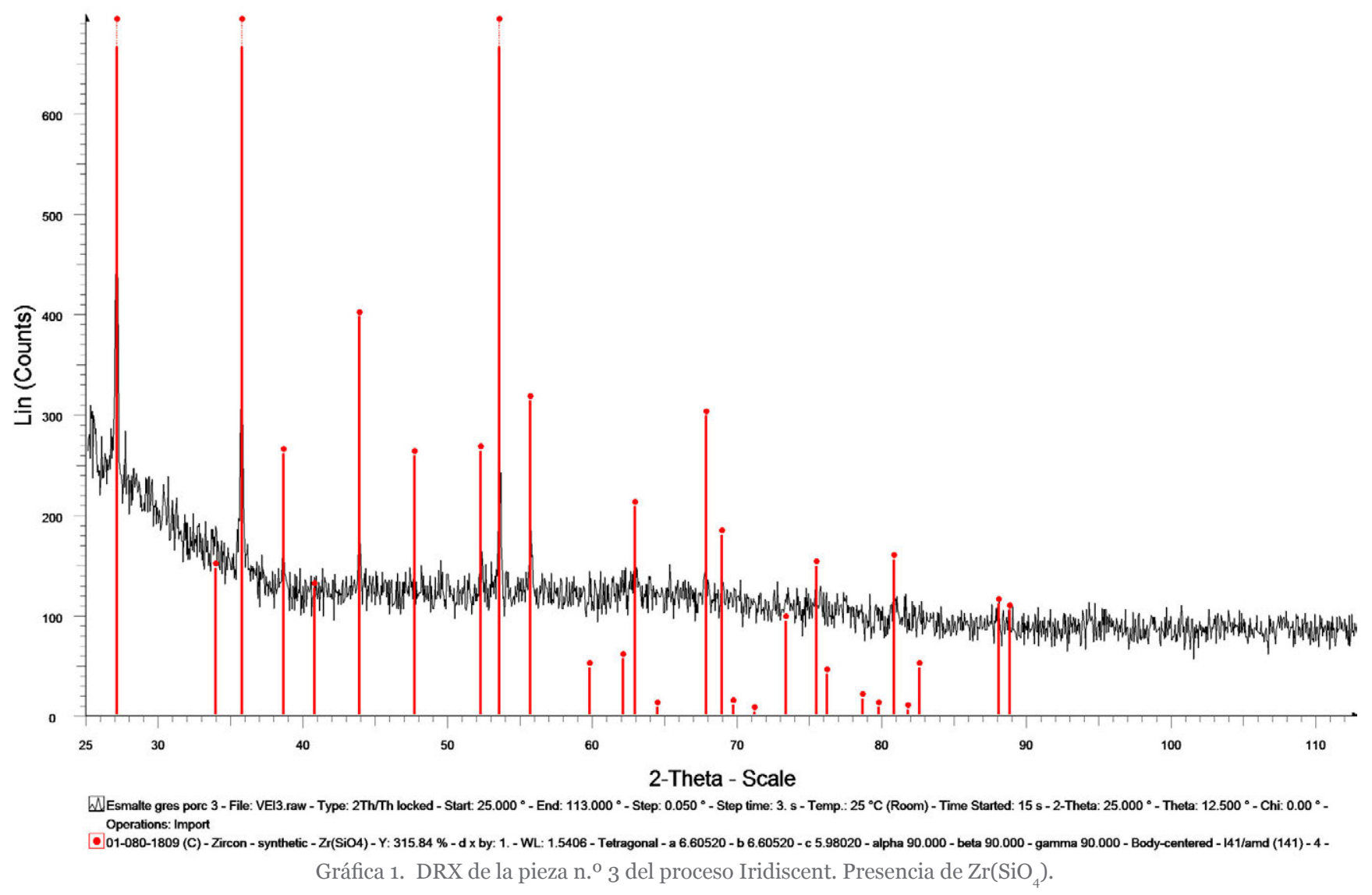

ción del silicato de zirconio favorece el efecto de difracción de la luz solar produciendo en mayor medida el efecto iridiscente.

Los resultados de las muestras 1, 2, 3 y 4 presentan la presencia de los referidos metales zirconio, zinc y titanio, con variaciones de escasa importancia en los porcentajes. Hay que tener en cuenta que el análisis que efectúa el microscopio analiza un espesor en torno a 4 micras. Podemos deducir que en ellas está presente el ya referido esmalte a base de zirconio, titanio y zinc. Sin embargo, en la muestra n. ${ }^{\circ} 5$ se observa la presencia de anatasa en un elevado porcentaje, entre un
17 y un $20 \%$ del análisis (Tabla 1). En la Figura 7 se aprecia a 300 y 3.000 aumentos un cuarteado de esta última capa de esmaltado a base de anatasa, con un espesor entre 0,5 y 1 micra. Esta microfisuración se debe a las diferencias de coeficientes de expansión térmica entre el titanio y el zirconio (14), 1,3 y $0,3 \times 10^{-5} \mathrm{pul} / \mathrm{pul} /{ }^{\circ} \mathrm{C}$. En la parte de la fisuración se observa que el esmalte inferior es exactamente similar a los presentados en las muestras 1 a 4 . La anatasa, en su aplicación final, refuerza enormemente el efecto de irisado de reflexión de la luz tanto por sus cualidades físicas como por su efecto de fisuración (15). Además se observa que el esmalte anterior, a base de óxidos de zirconio, titanio y zinc, no ha su-

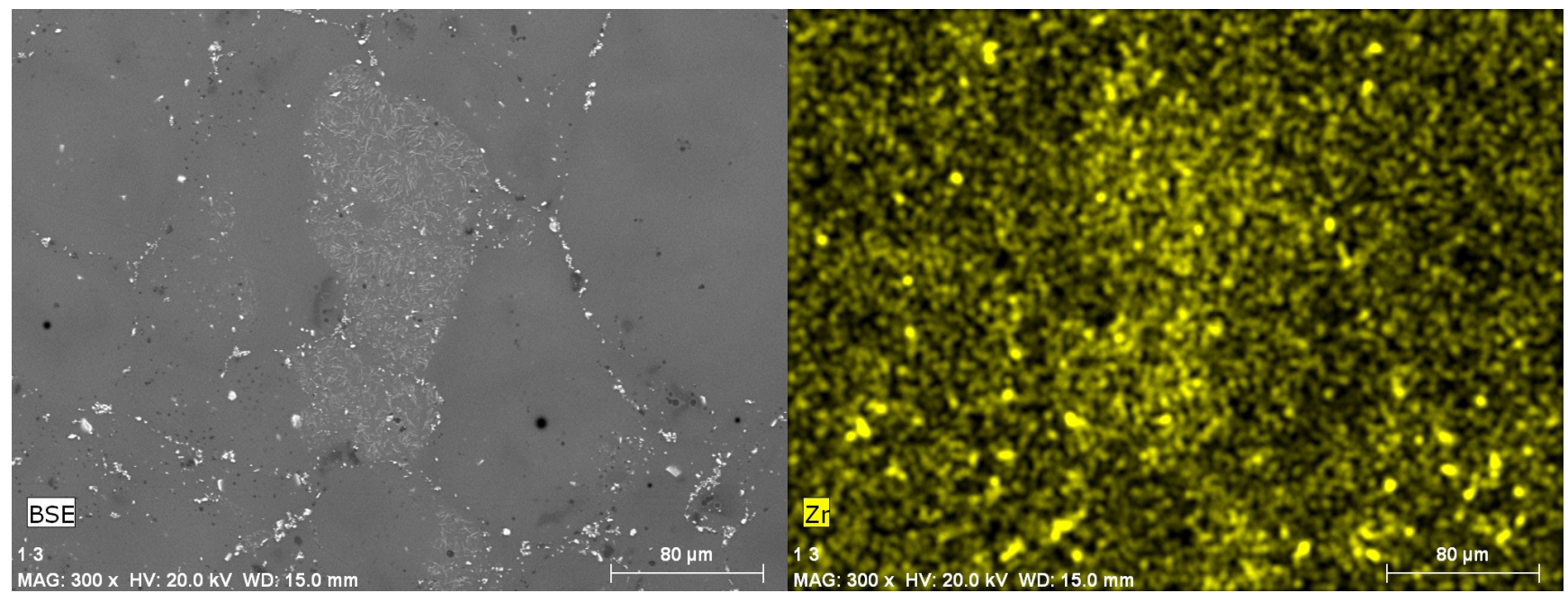

Figura 5. Microscopía electrónica de barrido a 300 aumentos. Presencia de zirconio. 


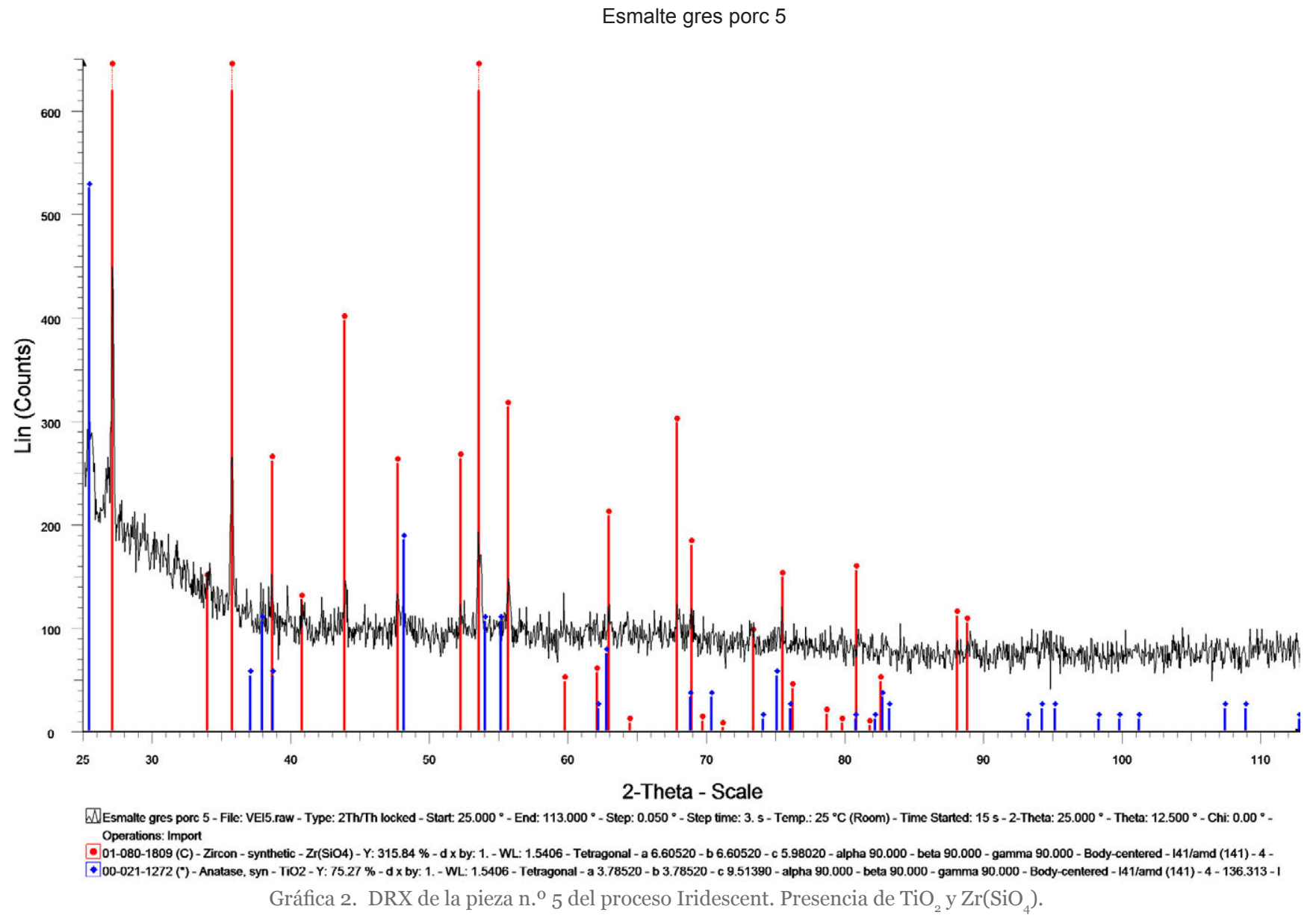

frido alteraciones ni tampoco microfisuración, conservando sus cualidades de protección de la pieza.

El resultado final del proceso de fabricación es una baldosa de gres porcelánico cuyas características constructivas son conocidas pero que incorpora un esmaltado vitrificado sin microfisuración, que le hace resistente frente a las acciones de la humedad, heladicidad y resistencia a acciones químicas, al tiempo que potencia las características de los colores goniocromáticos o iridiscentes que la componen.

La durabilidad del esmalte en este tipo de cerámica ha sido estudiada por diferentes autores tanto en productos por monococción como en doble o triple cocción. Las investigaciones de M. J. Cabrera sobre esmaltes de aspecto metálico en baldosas de monococción dieron como resultado una dureza al rayado en escala Mohs superior a 7 , un valor superior a otros esmaltes ricos en silicatos aplicados en soportes de gres y gres porcelánico (16); una resistencia a las manchas de clase 5 y una resistencia a la abrasión superficial PEI III (17). Cumplen así las resistencias exigidas por las normas UNE aplicables a las baldosas cerámicas.

Atendiendo a las características constructivas el cerámico Iridescent es un material de $10 \mathrm{~mm}$ de espesor, con capacidad de absorción de agua $0,1 \%$. Resistencia a flexión 52 $\mathrm{N} / \mathrm{mm}^{2}$ mayor de $35 \mathrm{~N} / \mathrm{mm}^{2}$; resistente a la abrasión superficial PEI V (18); resistente al cuarteo (19); resistente a la helada (20); resistente a agentes químicos: GHA (21); resistente a las manchas Clase 5 (22). Coeficiente fricción: ASTM fh $=0,39$, ASTM fs=0,63. Su baja absorción de agua implica la colocación mediante anclaje mecánico para evitar problemas de desprendimiento en gres porcelánico pegado en fachada (23).

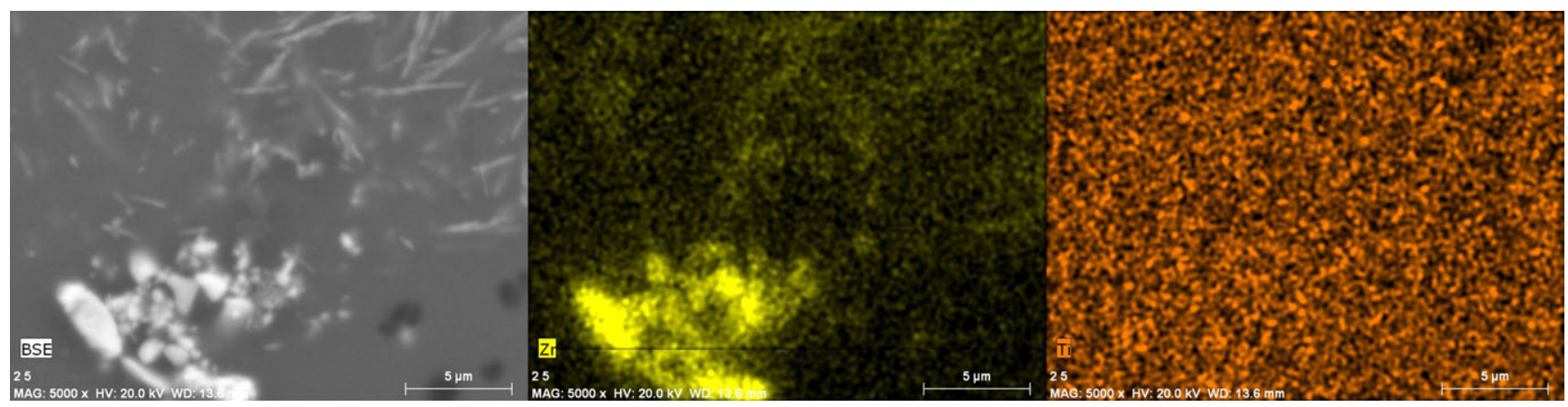

Figura 6. Muestra n. ${ }^{\circ}$ 2. Concentración de Zr en zonas. Reparto homogéneo del Ti. 5.00o aumentos. 
Tabla 1. Resultados de presencia de titanio, zirconio y zinc en las 5 muestras del proceso.

\begin{tabular}{|l|c|c|c|c|c|}
\hline & \multicolumn{5}{|c|}{ C norm. [wt.\%] } \\
\hline & MUESTRA 1 & MUESTRA 2 & MUESTRA 3 & MUESTRA 4 & MUESTRA 5 \\
\hline $\mathrm{O}$ & 44,79 & 45,40 & 43,13 & 39,28 & 38,47 \\
\hline $\mathrm{Mg}$ & 1,12 & 1,37 & 1,12 & 1,04 & 0,89 \\
\hline $\mathrm{Al}$ & 4,59 & 5,14 & 4,77 & 4,81 & 3,66 \\
\hline $\mathrm{Si}$ & 24,85 & 25,55 & 25,05 & 25,23 & 18,83 \\
\hline $\mathrm{K}$ & 2,40 & 2,26 & 2,41 & 2,27 & 1,91 \\
\hline $\mathrm{Ca}$ & 11,83 & 11,17 & 12,17 & 11,53 & 9,20 \\
\hline $\mathrm{Ti}$ & 1,67 & 1,33 & 3,58 & 7,14 & 20,77 \\
\hline $\mathrm{Zn}$ & 5,53 & 4,62 & 4,66 & 5,28 & 4,03 \\
\hline $\mathrm{Zr}$ & 3,23 & 3,16 & 3,12 & 3,41 & 2,25 \\
\hline TOTAL & $\mathbf{1 0 0 \%}$ & $\mathbf{1 0 0 \%}$ & $\mathbf{1 0 0 \%}$ & $\mathbf{1 0 0 \%}$ & $\mathbf{1 0 0 \%}$ \\
\hline
\end{tabular}

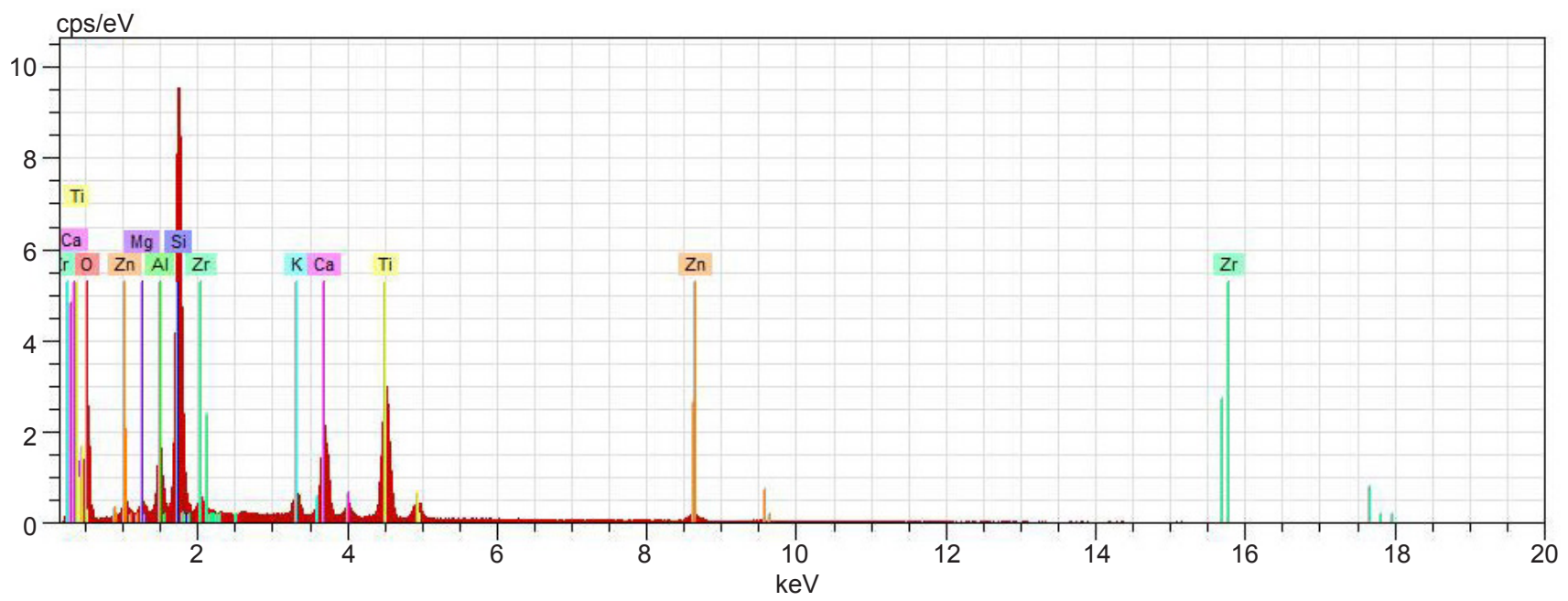

Gráfica 3. Muestra.$^{\circ}$ 5. Presencia de titanio en valores del $20 \%$ en las últimas 4 micras.

\section{ESTUDIO COLORIMÉTRICO}

A continuación evaluaremos los colores goniocromáticos, o iridiscentes, que presenta la cerámica Iridescent debido al proceso de curado térmico inhomogéneo descrito. El estudio colorimétrico se ha realizado principalmente usando un espectrofotómetro multi-ángulo, marca y modelo BYK-Gardner BYK-mac, consistente en una configuración de medida de la luz reflejada en varias direcciones de incidencia y recogida de la luz, o geometrías de medida, que se ajustan a la normativa
ASTM E2194, típicamente usada en los colores goniocromáticos para el sector automoción, pero también en cosmética y otros sectores industriales (24).

De una baldosa cerámica en fase inicial (muestra 1) de precurado térmico, y de otra en su fase final (muestra 5), se reconocieron básicamente unos colores característicos, que fueron finalmente los medidos. Las medidas de factor de radiancia espectral, o reflectancia, por tipo de geometría, y con el consiguiente análisis colorimétrico en el espacio CIE-

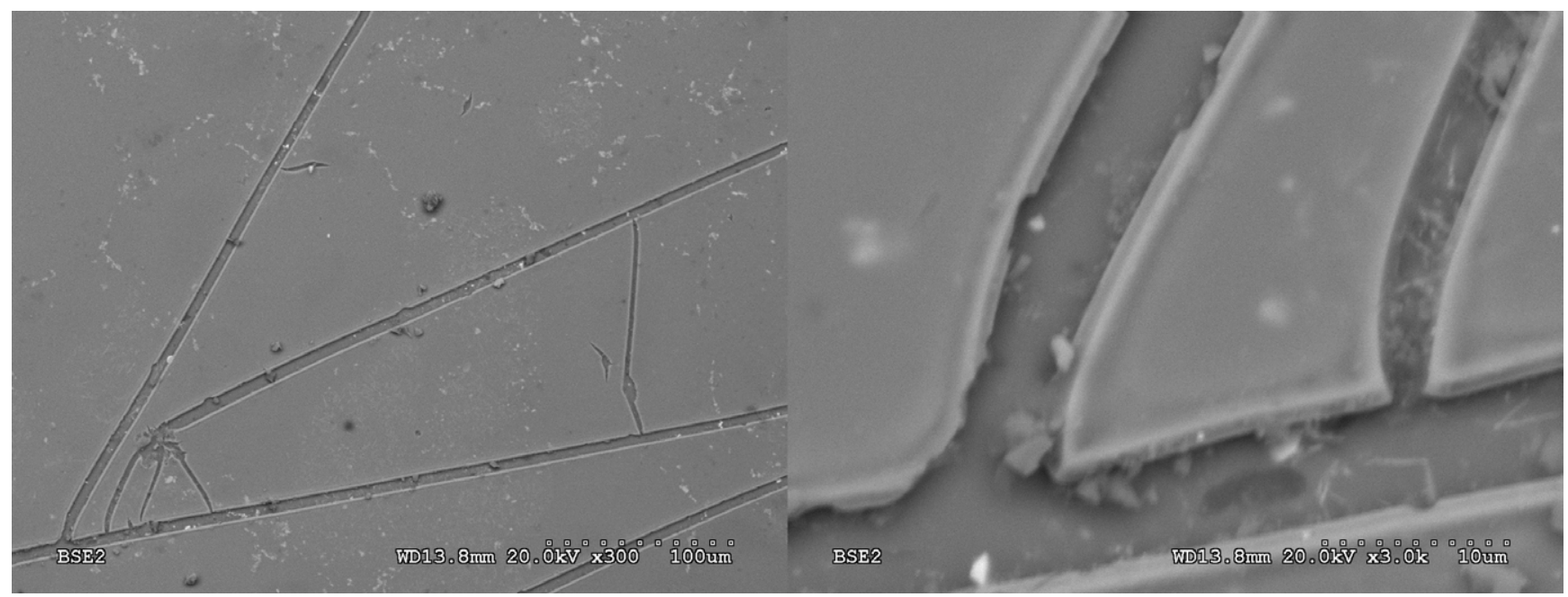

Figura 7. Muestra n. ${ }^{\circ}$ 5. Cuarteado de la capa final de anatasa a 300 y 3.000 aumentos. 


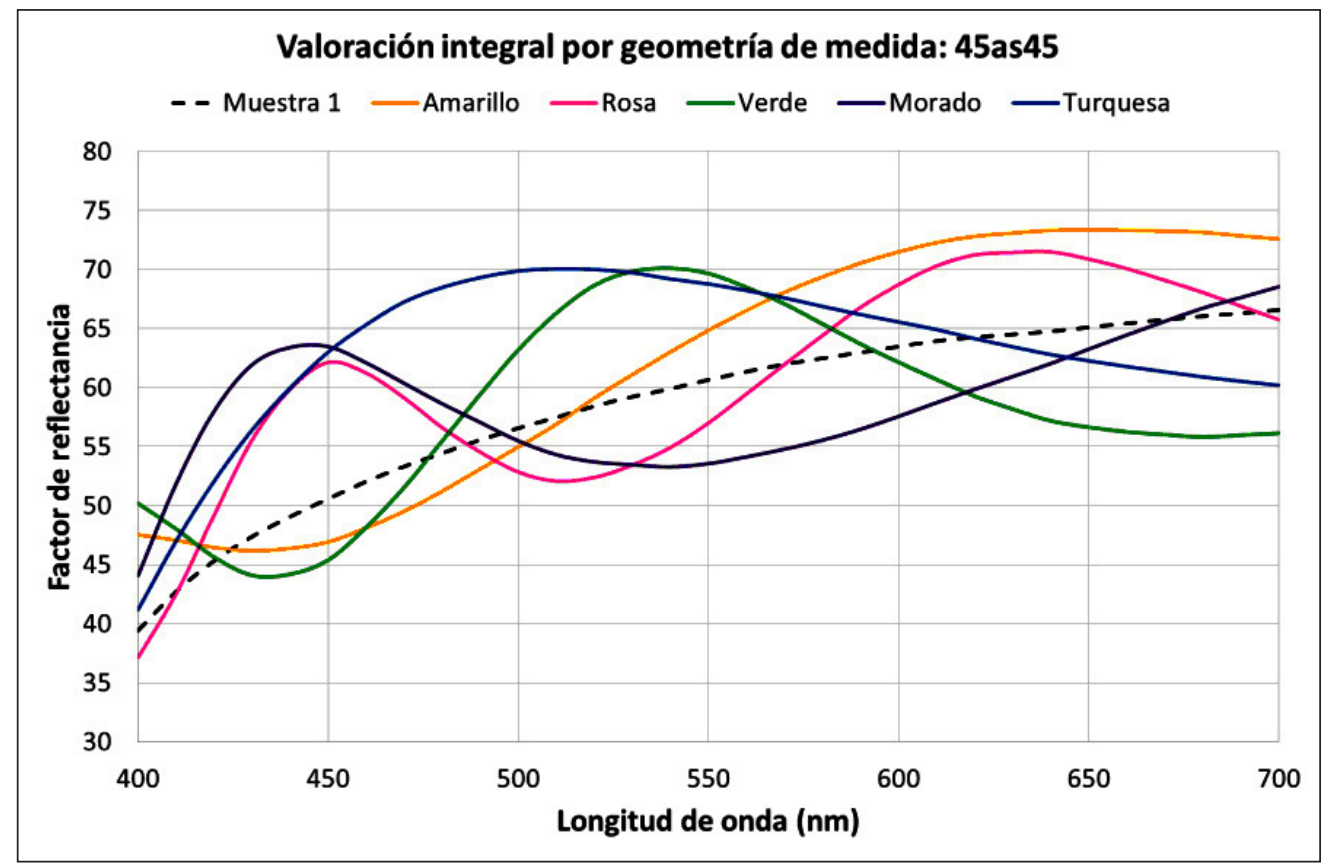

Gráfica 4. Curvas de reflectancia espectral de los colores característicos de la baldosa cerámica del MUCA para la geometría 45 as 45 .

$\mathrm{L}^{*} \mathrm{a} \mathrm{b}^{*}$ bajo iluminante D65 (luz diurna), muestran que el proceso de curado térmico proporciona cambios bruscos de tono, colorido y claridad con respecto a la muestra original (o muestra 1). Así, las medidas espectrales de los colores característicos (25) aparecen basculando alrededor del perfil espectral de la muestra 1 (Gráfica 4). Y, con el análisis colorimétrico en CIE-L* ${ }^{*}{ }^{*} b^{*} C^{*}{ }_{a b} h_{a b}$ (Figuras 8 y 9), se aprecia claramente estos cambios anunciados, que se correlacionan con lo percibido directamente.

Aparte de este análisis grupal de los colores característicos, y agrupando las medidas reflectancia por las seis geometrías en cada color característico, se comprobó que tras el proce- so de curado térmico estos colores (y, por consiguiente, sus pigmentos de efecto) presentan cierto grado de gonicromatismo individual, pero no tan elevado con respecto a los colores goniocromáticos (26) usados actualmente en pinturas para automóvil (27), cosmética, etc.

Los colores característicos y presentes simultáneamente en la misma baldosa cerámica, al conocer su caracterización espectral y colorimétrica para varias geometrías de medida representativas, pueden aportar una variedad cromática mucho más amplia, que podría observarse en este edificio con iluminación artificial en posiciones y direcciones concretas, lo cual le proporcionaría al edificio, y a la ciudad de La Al-

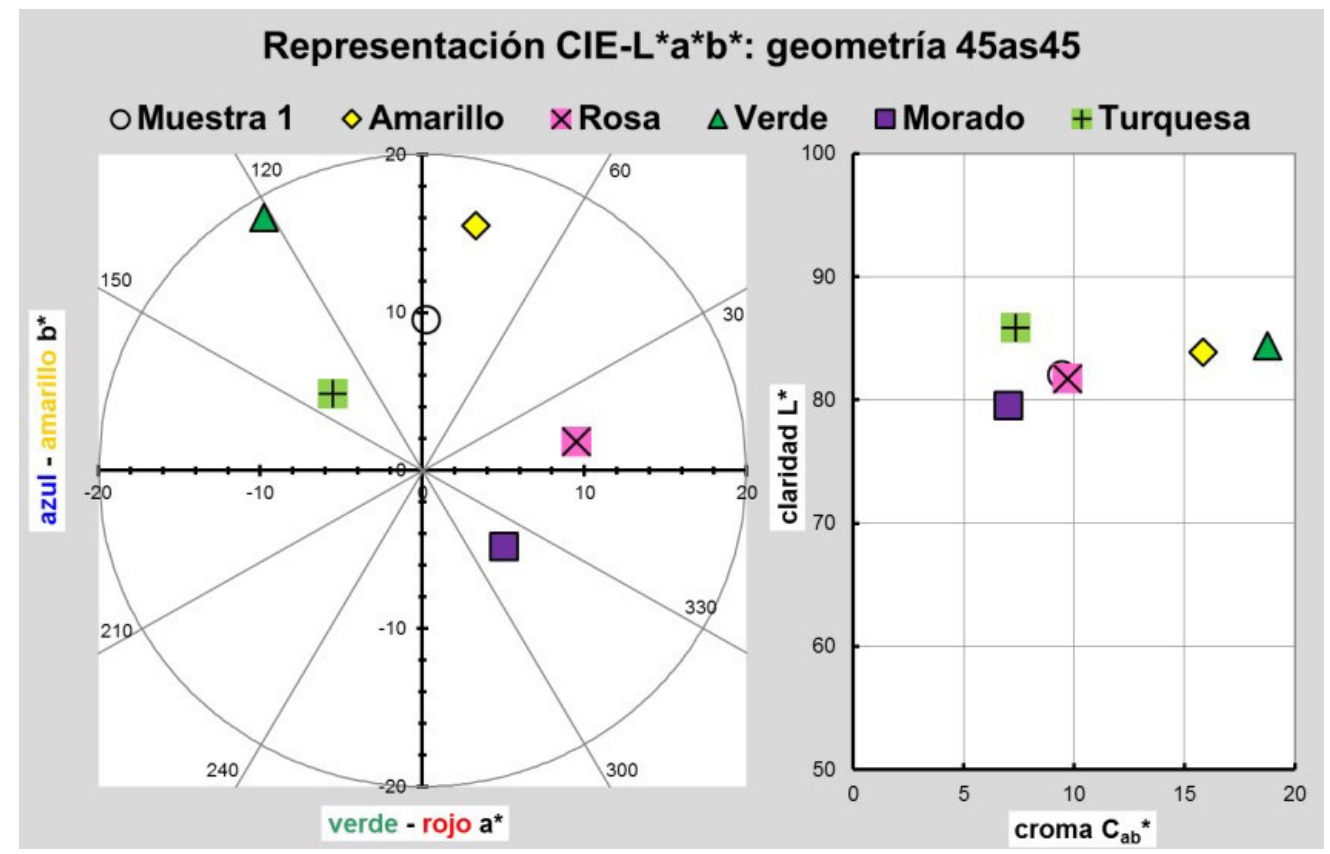

Figura 8. Representación gráfica en CIE-L*a*b* de los colores característicos de la fachada del MUCA en la geometría 45 as 45 . 


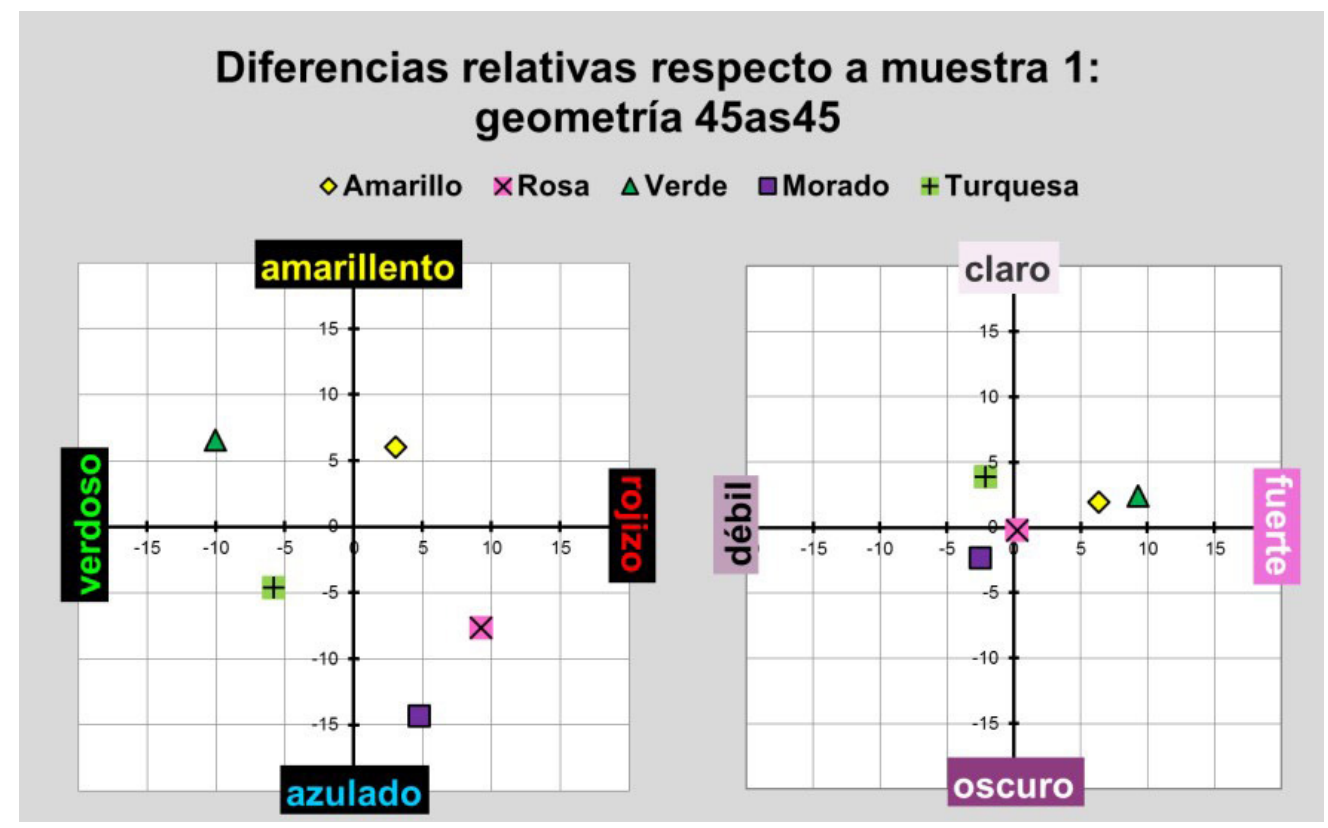

Figura 9. Representación gráfica en CIE- $\mathrm{L}^{*} \mathrm{a} * \mathrm{~b} *$ de los colores característicos de la fachada del MUCA en la geometría 45 as 45 .

güeña, un valor adicional del atractivo inicial de este edificio emblemático en el interior de la provincia de Alicante.

\section{CONCLUSIONES}

Son numerosas las investigaciones que se han desarrollado en los últimos años para abrir nuevos campos de aplicación de los materiales cerámicos en edificación. El gres porcelánico por prensado o extrusión, de formato habitual o de gran formato, se ha aplicado en envolventes de edificios aportando soluciones que no requieren mantenimiento y mejoran la eficiencia energética, al tiempo que aportan una gran calidad de diseño. Sin embargo, son escasas las investigaciones llevadas a cabo en el tratamiento del color y la reflexión de la luz.

La aplicación de esmaltes a base de óxidos de zirconio, titanio y zinc producen un efecto de irisado y nacarado que aportan valor a las envolventes, produciendo en el espectador unas novedosas características visuales y perceptivas de la arquitectura. La aplicación de forma artesanal de estos esmaltes produce diferentes efectos de reflexión de la luz, pudiéndose trabajar en líneas de obtención de diversas gamas cromáticas en función del proceso. El curado de vitrificado de estos esmaltes es crítico a la hora de obtener los resultados de irisado y nacarado. La capa de esmalte intermedia a base de los óxidos metálicos señalados no sufre fisuración si se hace un buen curado, ejerciendo así el esmalte funciones adecuadas de protección frente a ataques químicos y heladicidad. De entre estos elementos es el zirconio el que favorece de forma principal el efecto por su concentración en microzonas. El zinc y el titanio se distribuyen homogéneamente. Una última capa de óxido de titanio, tras una ulterior cocción a menor temperatura, refuerza el efecto pretendido debido en gran medida al efecto de microfisuración producido en ésta.

Como conclusión final, la fachada del MUCA presenta un atractivo visual gonicromático por el proceso de curado térmico espacialmente inhomogéneo de la baldosa cerámica preformulada inicialmente. Si bien, los colores finales o característicos, debido a la aparición de diferentes tipos de pigmentos inorgánicos goniocromáticos (con estructuras físicoópticas ligeramente diferentes), presentan un ligero grado de goniocromatismo o efecto metalizado, pero no tan impactante si se observaran de forma individual en una baldosa toda ella del mismo color.

\section{AGRADECIMIENTOS}

Nuestro agradecimiento a Francisco Miguel Martínez Verdú, Grupo de Visión y Color de la Universidad de Alicante. http://web.ua.es/gvc. Instituto Universitario de Física Aplicada a las Ciencias y Tecnologías (IUFACyT).

\section{REFERENCIAS}

(1) Pavimentos y revestimientos cerámicos. Innovación, vanguardia y sostenibilidad en espacios públicos y privados. Reportaje. [En línea] http://www.promateriales.com/pdf/pm2209.pdf

(2) Vacca, M.C. (2010). Recubrimiento de superficies arquitectónicas con piezas cerámicas, p. 159. Tesis doctoral. Barcelona: Universidad Politécnica de Cataluña.

(3) Puente, M. (2006). Conversaciones con Mies van der Rohe. Barcelona: Gustavo Gili.

(4) Cumella, A. Cerámica para la arquitectura. Granollers. [En línea] http://www.decorativa.es/recursos/docs/PROYECTOS.pdf

(5) Ruiz Geli, E. (2009). Villa Nurbs: Ceràmica i Arquitectura. Catálogo dedicado al proceso de construcción del edificio cerámico Villa Nurbs. Barcelona: Museu de Ceràmica. 
(6) Durán, J. Memoria del proyecto Color Revolution. Remodelación de la calle San Vicente en Burriana (Castellón). [En línea] http://www.premiosceramica.com/premiados.aspx?lang=es-ES\&tipo=arquitectura-interiorismo\&edicion=anteriores\&categoria $=$ premiados\&anyo $=2007 \& i d=53$

(7) Colomban, P. (2004). Secrets retrouvés du Lustre Abbasside. Revue Céramique \& Verre, n. ${ }^{\circ}$ 139, novembre/décembre. [En línea] http://www.glvt-cnrs.fr/ladir/pages/colomban/lustreceramique.pdf

(8) Nebot-Díaz, I. (2001). Nuevas tecnologías para el sector cerámico de Castellón: desarrollo de esmaltes vitrocristalinos y vitrocerámicos. Castellón: Universitat Jaume I.

(9) Pastor, J.Y., Poza, P., Llorca, J., Peña, J.I., Merino, R.I., Orera, V.M. (2001). Mechanical properties of directionally solidified $\mathrm{Al}_{2} \mathrm{O}_{3}-\mathrm{ZrO}_{2}\left(\mathrm{Y}_{2} \mathrm{O}_{3}\right)$ Eutectics. Materials Science and Engineering: A, 308: 241-249.

(10) Peña, J.I., Merino, R.I., de la Fuente, G.F., Orera, V.M. (1996). Aligned $\mathrm{ZrO}_{2}$ (c)-CaZrO eutectics grown by the laser floating zone method: electrical and optical properties. Advanced Materials, 8(11): 909-912.

(11) Merino, R.I. (2006). Cerámicas eutécticas solidificadas direccionalmente para fotónica y electrocerámica. Rev. Real Academia de Ciencias, 61: 47-86.

(12) Perla, A. (2004). La Cerámica, el reflejo del sol. Informes de la Construcción, 39(389): 55-59. [En línea] http://informesdelaconstruccion.revistas.csic.es/index.php/informesdelaconstruccion/article/view/1664/1843

(13) Recubrimiento para cuerpos cerámicos. Patente $n .^{\circ}$ solicitud 201230096. Titular Vidres, S. A.

(14) Vielhaber, L. (1961). Tecnología de los Esmaltes, p. 24. Barcelona: Editorial Reverté.

(15) Cabrera, M.J., Montins, V., Solsona, D., Sala, J.M. (2012). Obtención de efectos físico-ópticos para la decoración de baldosas cerámicas. Cerámica y Vidrio, marzo-abril: IX-XVI.

(16) Martínez, J., et al. (2004). Desarrollo de nuevos esmaltes para soportes de gres y gres porcelánico adaptados a los procesos de pulido y que presentan buenas prestaciones técnicas y estéticas. En Congreso Qualicer 2004 (pp. 137-152). Castellón: Cámara Oficial de Comercio, Industria y Navegación.

(17) Cabrera, M.J., Montíns, V., Foó, A., Balfagón, P. (2006). Obtención de esmaltes de aspecto metálico en baldosas fabricadas por monococción. En Congreso Qualicer 2006 (pp. 261-274). Castellón: Cámara Oficial de Comercio, Industria y Navegación.

(18) AENOR-CEN (1999). UNE-EN ISO 10545-7. Baldosas cerámicas. Parte 7: Determinación de la resistencia a la abrasión superficial de las baldosas esmaltadas. (ISO 10545-7:1996). Asociación Española de Normalización (AENOR).

(19) AENOR-CEN (1997). UNE-EN ISO 10545-11. Baldosas cerámicas. Parte 11: Determinación de la resistencia al cuarteo de baldosas esmaltadas. (ISO 10545-11:1994). Asociación Española de Normalización (AENOR).

(20) AENOR-CEN (1997). UNE-EN ISO 10545-12. Baldosas cerámicas. Parte 12: Determinación de la resistencia a la helada. (ISO 10545-12:1995, incluye Corrigendum Técnico 1:1997). Asociación Española de Normalización (AENOR).

(21) AENOR-CEN (1998). UNE-EN ISO 10545-13. Baldosas cerámicas. Parte 13: Determinación de la resistencia química. (ISO 10545-13:1995). Asociación Española de Normalización (AENOR).

(22) AENOR-CEN (1998). UNE-EN ISO 10545-14. Baldosas cerámicas. Parte 14: Determinación de la resistencia a las manchas. (ISO 10545-14:1995, incluye Corrigendum Técnico 1:1997). Asociación Española de Normalización (AENOR).

(23) Delgado-Méndez, L., del Moral-Ávila, C., Valverde-Palacios, I., Valverde-Espinosa, I. (2015). Diagnosis de una patología en gres porcelánico pegado en fachada. Informes de la Construcción, 67(539): e108, doi: http://dx.doi.org/10.3989/ ic.14.085.

(24) ASTM International. (2014). ASTM E2194-14, Standard Test Method for Multiangle Color Measurement of Metal Flake Pigmented Materials. West Conshohocken, PA: ASTM International, http://www.astm.org.

(25) Ferrero, A., Perales, E., Rabal, A., Campos, J., Martínez-Verdú, F.M., Chorro, E., Pons, A. (2014). Color representation and interpretation of special effect coatings. The Journal of the Optical Society of America A, 32(2): 436-447.

(26) Klein, G.A. (2010). Industrial color physics. New York: Springer.

(27) Chorro, E., Perales, E., Burgos, F.J., Gómez, O., Vilaseca, M., Viqueira, V., Pujol, J., Martínez-Verdú, F.M. (2015). The minimum number of measurements for colour, sparkle, and graininess characterisation in gonio-apparent panels. Coloration Technology, 131(4): 303-309. 